\title{
PARALLEL REFLECTION OF LIGHT BY PLANE MIRRORS*
}

\section{BY JOSEPH B. KELLER (Institute for Mathematics and Mechanics, New York University)}

1. Introduction. If three plane mirrors are mutually orthogonal then, as is well known in geometrical optics, any ray ${ }^{1}$ incident on these mirrors in the octant which they bound will be reflected once from each mirror and will emerge parallel to its original direction. Similarly any ray incident on two orthogonal plane mirrors, in the quadrant which they bound and in the plane perpendicular to them, will be reflected once from each mirror and will emerge parallel to its original direction. It is interesting to investigate whether there are other configurations of plane mirrors which also have this property of parallel reflection.

For two mirrors, with the incident ray in the plane perpendicular to them, the answer is easily found although it does not seem to be well known. It is this: if and only if the mirrors meet at an angle $\pi / n$, where $n$ is any even integer, every ray will emerge parallel to its original direction. Furthermore every ray suffers $n$ reflections; for example, two in the case of orthogonal mirrors discussed above.

For three mirrors meeting at a point Synge ${ }^{2}$ has shown that if every ray is reflected exactly once from each mirror every ray will emerge parallel to its original direction if and only if the mirrors are mutually orthogonal. However, in view of our above result for two mirrors, it is reasonable to drop the restriction that every ray be reflected exactly once from each mirror. We then find that there are indeed other configurations of three mirrors meeting at a point which yield parallel reflection for every incident ray. These are the configurations in which the sets of dihedral angles are $(\pi / 2, \pi / 3, \pi / 4),(\pi / 2, \pi / 3, \pi / 5)$ and $(\pi / 2, \pi / 2, \pi / n)$ where $n$ is an even integer. The numbers of reflections suffered by any ray are 9,15 and $n+1$ respectively. The case $(\pi / 2, \pi / 2, \pi / n)$ with $n=2$ is the case of mutually orthogonal mirrors, in which case each ray is reflected three times. Furthermore, these are the only configurations of three or more mirrors meeting at a point which have the parallel reflection property.

In section II we formulate the problem, and in section III we deduce the result described above.

2. Formulation. Suppose three planes meet at a single point and that one side of each plane is designated as the reflecting side. Then one and only one of the eight regions into which the planes divide space will be on the reflecting side of all three mirrors. Any oriented straight line in this region which can be extended indefinitely in its negative direction without intersecting a plane is called an incident ray ${ }^{1}$. If an incident ray intersects a plane it is reflected according to the law of reflection; the reflected ray may be again reflected, etc. If a reflected ray can be extended indefinitely in its positive direction

*Received June 2, 1952. This work was performed at Washington Square College of Arts and Science, New York University and was supported in part by Contract No. AF-19(122)-42 with the U.S. Air Force through sponsorship of Geophysics Research Division, Air Force Cambridge Research Center, Air Materiel Command.

${ }^{1}$ Rays which intersect an edge or vertex or are parallel to one or two mirrors are excluded. The latter rays do emerge parallel to their original directions although they suffer only two or one reflections, respectively, not being reflected from the mirrors to which they are parallel.

2J. L. Synge, Reflection in a Corner Formed by Three Plane Mirrors, Quarterly of Applied Math., 4, $166-176$ (1946). 
without intersecting a plane, it is called a final reflected ray. The problem we consider is that of finding all configurations of three planes meeting at a point with the property that every final reflected ray is parallel to the incident ray from which it originates.

First, following Synge, we shall reformulate the problem in terms of reflection of points on the surface of a sphere. To this end we construct a sphere with center at the meeting point of the planes. The surface of the sphere intersects the planes in great circles. The trihedral region on the reflecting sides of all three planes cuts out on the sphere a spherical triangle $T$, which is bounded by the three great circles. We represent a ray by the point of intersection, with the sphere, of a parallel infinite line through the center. Since a straight line through the center intersects the sphere twice the more negative of the two points is chosen. Thus all parallel rays with the same orientation are represented by the same point, while antiparallel rays are represented by antipodal points. It is clear that all incident rays, defined above, are represented by points in the spherical triangle $T$.

Now suppose that a ray, represented by the point $P$, is reflected in a plane, represented by the great circle $A$. Then the reflected ray is represented by a point $P_{1}$ which is obtained by reflecting $P$ in $A$. This can easily be seen if the point of reflection is transported to the center of the sphere. The ray from $P$ to the center is the incident ray; the ray from $P_{1}$ to the center is the negative extension of the reflected ray.

Since, by definition, only one side of each plane is reflecting a point $P$ must lie on the reflecting side of a great circle $A$ in order to be reflectable in $A$. We will call a reflection of $P$ in $A$ admissible if $P$ does lie on the reflecting side of $A$. The reflecting side of $A$ is the side or hemisphere containing the spherical triangle $T$, according to the definition of $T$. Now a ray emerges, or is a final reflected ray in accordance with the definition above, only when it is not reflectable in any plane. Thus a point $P$ represents a final reflected ray only if it lies on the non-reflecting side of each plane. But every point which lies in all three hemispheres not containing $T$ must lie in the spherical Triangle $T^{\prime}$ antipodal to $T$. We can now formulate our problem as that of finding all spherical triangles $T$ with the property that every sequence of admissible reflections of any point $P$ in $T$ leads to the antipodal point $P^{\prime}$ in $T^{\prime}$, since $P^{\prime}$ represents a final ray parallel to the incident ray represented by $P$.

3. Solution. Let us suppose that $T$ is a triangle with the desired property and that $P_{1}$ and $P_{2}$ are two points in $\mathrm{T}$. We first want to prove that if an admissible sequence of reflections $R_{1}$ carries $P_{1}$ into some point $P$, and an admissible sequence of reflections $R_{2}$ carries $P_{2}$ into the same point $P$, then $P_{1}$ and $P_{2}$ are identical. In other words, no two distinct points can have a common image under admissible sequences of reflections. To prove this assertion we note that, by hypothesis, every sequence of admissible reflections of a point of $T$ terminates on the antipodal point. Thus, in particular, the sequence $R_{1}$ which carries $P_{1}$ into $P$ can be continued by a sequence of admissible reflections which we shall call $S_{1}$ so that $R_{1}+S_{1}$ carries $P_{1}$ into $P_{1}^{\prime}$, the antipodal point. But then $S_{1}$ carries $P$ into $P_{1}^{\prime}$. Therefore $R_{2}+S_{1}$ carries $P_{2}$ through $P$ into $P_{1}^{\prime}$. Since this is an admissible sequence of reflections, it must by hypothesis terminate on $P_{2}^{\prime}$. Therefore $P_{1}^{\prime}$ is identical with $P_{2}^{\prime}$ and so $P_{1}$ is identical with $P_{2}$.

Now let us consider the neighborhood of a vertex of $T$ of vertex angle $\alpha$. We wish to consider successive reflections of a point of $T$ in the two sides meeting at the vertex. We introduce polar coordinates with the vertex as origin and one side as polar axis. If $\phi$ is the polar angle the other side is given by $\phi=\alpha$. If $\phi=\theta$ is the angular coordinate 
of a point of $T$, its image after $n$ successive reflections in the sides 0 and $\alpha$ will have the coordinate $\phi_{n}(\theta)$ given by:

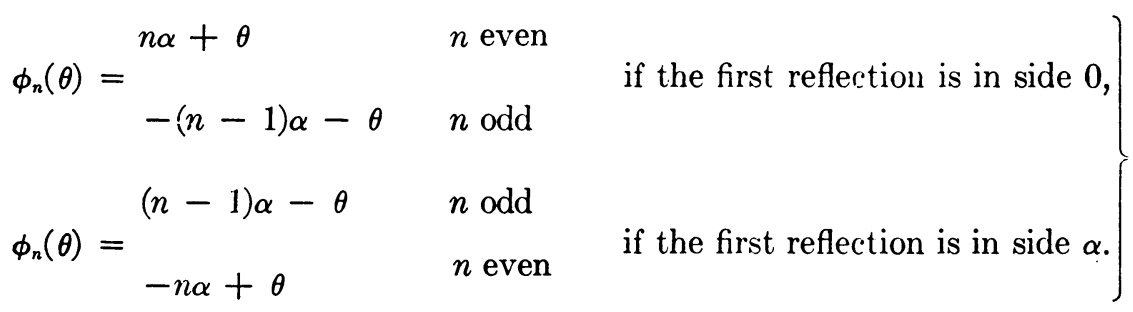

These reflections are admissible only until the image falls in the vertical angle $\pi$ to $\pi+$ $\alpha$ (or $-\pi$ to $-\pi+\alpha$ ) since then the image is on the non-reflecting side of both mirrors meeting at the vertex. [To prove the result stated previously for two mirrors, we set $\phi_{n}=\pi+\theta$ or $-\pi+\theta$ and find $\alpha=\pi / 2$ with $n$ even.]

From the expressions in Eq. 1 it is not difficult to show that some image of every point will lie in the vertical angle $\pi$ to $\pi+\alpha$. Let us consider the least value of $n$ for which the image of any point of the original angle lies in the vertical angle. The sequence of admissible reflections which carries a particular point into the vertical angle in this number of reflections is necessarily an admissible sequence for all points in the original angle. Therefore the image of the original angular region under this sequence is another angular region which overlaps the vertical angle. If this overlapping is complete, then one may deduce, as above, that $\alpha=\pi / n$ where $n$ is an odd or even integer. If the overlapping is incomplete the image covers an edge of the vertical angle. The points outside this edge may then be reflected in it, and some of them will fall on already covered points. Therefore distinct points of the original angle will have a common image, contradicting the theorem above. Thus the overlapping must be complete and therefore, we conclude that each vertical angle is of the form $\pi / n$, where $n$ is an integer, not necessarily the same for all vertices.

Combining this result with the fact that the sum of the interior angles of a spherical triangle exceed $\pi$, we obtain the inequality.

$$
\frac{\pi}{n_{1}}+\frac{\pi}{n_{2}}+\frac{\pi}{n_{3}}>\pi
$$

The only solutions of this inequality are:

$$
\left(\frac{\pi}{2}, \frac{\pi}{3}, \frac{\pi}{3}\right), \quad\left(\frac{\pi}{2}, \frac{\pi}{3}, \frac{\pi}{4}\right), \quad\left(\frac{\pi}{2}, \frac{\pi}{3}, \frac{\pi}{5}\right), \quad\left(\frac{\pi}{2}, \frac{\pi}{2}, \frac{\pi}{n}\right) .
$$

Thus the only possible triangles having the desired property are included here since these solutions were obtained by imposing necessary conditions.

By examining the admissible reflections of these triangles on a sphere, we find that only the following actually do yield parallel reflection:

$$
\left(\frac{\pi}{2}, \frac{\pi}{3}, \frac{\pi}{4}\right), \quad\left(\frac{\pi}{2}, \frac{\pi}{3}, \frac{\pi}{5}\right), \quad\left(\frac{\pi}{2}, \frac{\pi}{2}, \frac{\pi}{n}\right), n \text { even. }
$$

The other triangles do map onto their antipodal triangles but with the sides interchanged; in other words, a point does not land on its antipode but on the antipode of its image in the principal altitude of the triangle. In the same process we find that the respective 
numbers of reflections are $9,1 j$ and $n+1$. This completes the proof for the case of three mirrors.

For more than three mirrors all the considerations preceding equation 2 are valid, but the corresponding inequality has no integer solutions at all.

To prove only Synge's result, which is included above, a much simpler method suffices. If a point arbitrarily near a vertex is reflected successively once in each of the sides meeting there the image remains arbitrarily close to the vertex. The third reflection must bring it to the antipode, an angular distance arbitrarily close to $\pi$ radius. Thus the angular distance from the vertex to the third side is arbitrarily near $\pi / 2$, and this applies to each side. Thus the triangle must be an octant of a sphere, which completes the necessity proof. The sufficiency is proved by reflecting an octant three times in its sides.

It is of interest to compare our results with those described on pps. 81-83 of Coxeter's "Regular Polytopes" (Methuen, London, 1948). There the discrete groups of reflections generated by three or more concurrent planes are deduced. It is first found that all the dihedral angles must be of the form $\pi / n$, then equation 2 is applied, and the solutions are given by equation 3 , with none for more than three planes. In the problem of discrete groups all sequences of reflections are permitted, while in the present problem only "admissible" sequences of reflections are permitted, which is a weaker condition. On the other hand we have the condition that some admissible sequence must carry each point into its antipode, and this condition is strong enough to limit the solutions to those given in equation 4-a subset of the configurations generating discrete groups.

The new parallel reflecting configurations given herein may have practical value in some applications because they reflect back only those rays which are incident from a certain angular region, and the angular region may be made much smaller than that of the mutually orthogonal mirrors.

I wish to express my indebtedness to my colleague, Prof. Wilhelm Magnus, who supplied a crucial part of the necessity proof given above.

\section{NOTE ON SELF-PROPAGATION OF TURBULENT SPOTS*}

\section{BY CARL E. PEARSON (Harvard University)}

Introduction. Emmons (1), (2) has recently directed attention towards the manner in which transition occurs from a laminar to a turbulent boundary layer. It appears that the dominating phenomenon is the spontaneous generation of a large number of turbulent "spots" which grow rapidly and eventually coalesce. It has been observed experimentally that, except perhaps immediately after generation, each spot grows in such a manner as to maintain geometric similarity. Because the spot is growing in a boundary laver, the rate at which an elemental portion of its periphery propagates its turbulence outwards is dependent on the orientation of that portion; this dependency of propagation rate on orientation is necessarily associated with the shape of the spot.

It is the purpose of this note to prove the simple but interesting result that for either of two reasonable speculative hypotheses concerning propagation rate, the shape of the spot approaches a certain asymptotic shape which is independent of the initial spot shape.

*Received July 18, 1952. 